Bull. Austral. Math. Soc.

VoL. 50 (1994) [49-53]

\title{
A NIL-IMPLIES-NILPOTENT RESULT IN LINEARLY COMPACT RINGS
}

\author{
WEIMIN XUE
}

\section{Dedicated to the memory of Professor I.N. Herstein}

Let $R$ be a left linearly compact ring with left ideals $I \subseteq J$ such that ${ }_{R} J$ is finitely generated and $R(J / I)$ is Artinian. We prove that if $J$ is nil over $I$ then $J$ is nilpotent over $I$.

\section{INTRODUCTION}

Let $R$ be an associative ring with identity. Let $I \subseteq J$ be left ideals of $R$ such that $J$ is nil over $I$, in the sense that for each $a \in J$ there exists a natural number $n=n(a)$ such that $a^{n} \in I$. Herstein [2] conjectured that if $R$ is left Noetherian then $J$ is nilpotent over $I$, that is, $J^{m} \subseteq I$ for some natural number $m$. Although this conjecture is still open, partial answers have been given in [2], [3], [5] and [6]. Meyer [5] verified Herstein's conjecture for left Artinian rings. This was also proved (but not published) independently by Herstein and Stafford, as mentioned in [3]. In this note, we consider Herstein's conjecture for left linearly compact rings instead of left Noetherian rings. Generalising the above mentioned result of Herstein, Meyer and Stafford, we shall establish the following

THEOREM 1. Let $R$ be a left linearly compact ring with left ideals $I \subseteq J$ such that ${ }_{R} J$ is finitely generated and ${ }_{R}(J / I)$ is Artinian. If $J$ is nil over $I$ then $J$ is nilpotent over $I$.

\section{PRELIMINARIES}

Basic properties of linearly compact modules are presented in Section 3 of the author's book [7]. Here we recall some definitions for the convenience of the reader.

Let ${ }_{R} M$ be a unitary left $R$-module. We use $N \leqq M$ to denote that $N$ is a submodule of $M$, and $M^{(n)}$ to denote the direct sum of $n$ copies of $M$. A family $\left\{m_{i}, M_{i}\right\}_{i \in I}$, where each $m_{i} \in M$ and $M_{i} \leqq M$, is called solvable in case there exists an $m \in M$ such that $m-m_{i} \in M_{i}$ for all $i \in I$; and it is called finitely solvable in

Received 21 September 1993

Copyright Clearance Centre, Inc. Serial-fee code: 0004-9729/94 \$A2.00+0.00. 
case $\left\{m_{i}, M_{i}\right\}_{i \in F}$ is solvable for each finite subset $F \subseteq I$. The module $M$ is called linearly compact in case each finitely solvable family of $M$ is solvable. For example, each Artinian module is linearly compact. The ring $R$ is called left linearly compact if the module ${ }_{R} R$ is a linearly compact $R$-module. Hence a left Artinian ring is both left Noetherian and left linearly compact, and our Theorem 1 is indeed a generalisation of the main result in [5].

For the sake of completness, we give the following result which is essentially due to Menini [4].

LEMMA 2. Let $J$ be a left ideal of a left linearly compact ring $R$. If ${ }_{R} M$ is a finitely generated $R$-module then $\left(\bigcap_{k=1}^{\infty} J^{k}\right) M=\bigcap_{k=1}^{\infty} J^{k} M$. In particular, if ${ }_{R} J$ is finitely generated then $\left(\bigcap_{k=1}^{\infty} J^{k}\right) J=\bigcap_{k=1}^{\infty} J^{k}$.

Proof: Let $M=\sum_{i=1}^{n} R m_{i}$ and let

$$
f: R^{(n)} \longrightarrow M \quad \text { via } \quad\left(r_{1}, \ldots, r_{n}\right) \longmapsto \sum_{i=1}^{n} r_{i} m_{i}
$$

Then $f$ is an $R$-module epimorphism. Now

$$
f\left(\bigcap_{k=1}^{\infty} J^{k} R^{(n)}\right)=f\left(\bigcap_{k=1}^{\infty}\left(J^{k}\right)^{(n)}\right)=f\left(\left(\bigcap_{k=1}^{\infty} J^{k}\right)^{(n)}\right)=\left(\bigcap_{k=1}^{\infty} J^{k}\right) M .
$$

On the other hand,

$$
\bigcap_{k=1}^{\infty} f\left(J^{k} R^{(n)}\right)=\bigcap_{k=1}^{\infty} J^{k} f\left(R^{(n)}\right)=\bigcap_{k=1}^{\infty} J^{k} M
$$

Since ${ }_{R} R^{(n)}$ is linearly compact, the equality $f\left(\bigcap_{k=1}^{\infty} J^{k} R^{(n)}\right)=\bigcap_{k=1}^{\infty} f\left(J^{k} R^{(n)}\right)$ holds by Leptin's Theorem (see, for example, [7, Theorem 3.8]). That is, we have $\left(\bigcap_{k=1}^{\infty} J^{k}\right) M=$ $\bigcap_{k=1}^{\infty} J^{k} M$

\section{Proof of Theorem 1}

Now we are ready to prove the main result.

Suppose $J$ is not nilpotent over $I$, that is, $J^{k} \nsubseteq I$ for each $k \in \mathbb{N}$. We shall derive a contradiction 
Since ${ }_{R}(J / I)$ is Artinian, there exists an $m \in \mathbb{N}$ such that $J^{m}+I=J^{m+k}+I$ for each $k \in \mathbb{N}$. Since ${ }_{R} R$ is linearly compact, by [7, Corollary 3.9], we have

$$
\left(\bigcap_{k=1}^{\infty} J^{k}\right)+I=\bigcap_{k=1}^{\infty}\left(J^{k}+I\right)=J^{m}+I \nsubseteq I .
$$

Hence $\bigcap_{k=1}^{\infty} J^{k} \nsubseteq I$. Let

$$
W=\left\{H / I \mid I \leqq H \leqq J \text { and }\left(\bigcap_{k=1}^{\infty} J^{k}\right) H \nsubseteq I\right\} .
$$

Since ${ }_{R} J$ is finitely generated, by Lemma 1 we have

$$
\left(\bigcap_{k=1}^{\infty} J^{k}\right) J=\bigcap_{k=1}^{\infty} J^{k} \nsubseteq I,
$$

so $J / I \in W$. Since ${ }_{R}(J / I)$ is Artinian, $W$ has a minimal element, say $H / I$. Then $\left(\bigcap_{k=1}^{\infty} J^{k}\right) H \nsubseteq I$ and $\left(\bigcap_{k=1}^{\infty} J^{k}\right) h \nsubseteq I$ for some $h \in H$. Since

$$
\left(\bigcap_{k=1}^{\infty} J^{k}\right) h \supseteq\left(\bigcap_{k=1}^{\infty} J^{k}\right) h^{2} \supseteq \ldots
$$

and $h$ is nil over $I$, there exists a $n \in \mathbb{N}$ such that $\left(\bigcap_{k=1}^{\infty} J^{k}\right) h^{n} \nsubseteq I$ and $\left(\bigcap_{k=1}^{\infty} J^{k}\right) h^{n+k} \subseteq I$ for each $k \in \mathbb{N}$. Without loss of generality, we assume that

$$
\left(\bigcap_{k=1}^{\infty} J^{k}\right) h \nsubseteq I \text { and }\left(\bigcap_{k=1}^{\infty} J^{k}\right) h^{k} \subseteq I \text { for each integer } k \geqq 2 .
$$

Since $\left(\bigcap_{k=1}^{\infty} J^{k}\right) J h=\left(\bigcap_{k=1}^{\infty} J^{k}\right) h \nsubseteq I$, we see that $(I+J h) / I \in W$. By the minimality of $H / I$, we have $I+J h=H$. Hence $j h=h+i$ for some $j \in J$ and $i \in I$. Then for each $k \in \mathbb{N}$, we have $j^{k} h=h+i_{k}$ for some $i_{k} \in I$. Since $J$ is nil over $I$, $j^{k} \in I$ if $k$ is sufficiently large. Hence we may assume that

$$
j \in I \text { and } j^{k} h=h+i_{k} \text { where } i_{k} \in I \text { and } k \in \mathbb{N} \text {. }
$$

Then, there exists an integer $n \geqq 2$ such that $(h+j)^{n} \in I$, since $j \in I$ and $h \notin I$. So,

$$
(h+j)^{n-1} h=h^{n}+j^{n-1} h+\sum \ldots h^{p} j^{k} h^{r}=h^{n}+h+i_{n-1}+\sum \ldots h^{p} j^{k} h^{r}
$$


is an element of $I$, where each of $p, k, r \in \mathbb{N}$.

Let $x=\ldots h^{p} j^{k} h^{r}$.

If $r \geqq 2, x=i h^{r}$ for some $i \in I$.

Now let $r=1$. Then

(i) $x=h^{p} j^{k} h=h^{p}\left(h+i_{k}\right)=h^{p+1}+i$ for some $i \in I$, where $p+1 \geqq 2$; or

(ii) $x=\ldots j^{q} h^{p} j^{k} h(q \in \mathbb{N})$. In this case, $x=\ldots j^{q}\left(h^{p+1}+i\right)=i^{\prime} h^{p+1}+i^{\prime \prime}$ for some $i^{\prime}, i^{\prime \prime} \in I$, where $p+1 \geqq 2$.

Hence $I$ contains an element

$$
\begin{aligned}
h^{n} & +h+\sum \ldots h^{p} j^{k} h^{r} \\
& =h^{n}+h+k_{2} h^{2}+\ldots+k_{n-1} h^{n-1}+i_{2}^{\prime} h^{2}+\ldots+i_{n-1}^{t} h^{n-1}+i_{n}^{\prime}
\end{aligned}
$$

where $k_{2}, \ldots, k_{n-1} \in \mathbb{N}$ and $i_{2}^{\prime}, \ldots, i_{n}^{\prime} \in I$. Hence we have

$$
\left(\bigcap_{k=1}^{\infty} J^{k}\right)\left(h^{n}+h+k_{2} h^{2}+\ldots+k_{n-1} h^{n-1}+i_{2}^{\prime} h^{2}+\ldots+i_{n-1}^{\prime} h^{n-1}\right) \subseteq I
$$

Since $n \geqq 2$, by (1) we get,

$$
\left(\bigcap_{k=1}^{\infty} J^{k}\right) h^{n} \subseteq I
$$

and

$$
\left(\bigcap_{k=1}^{\infty} J^{k}\right) k_{t} h^{t} \subseteq I
$$

for each $t=2, \ldots, n-1$. Since $\left(\bigcap_{k=1}^{\infty} J^{k}\right) J=\left(\bigcap_{k=1}^{\infty} J^{k}\right)$, we have

$$
\left(\bigcap_{k=1}^{\infty} J^{k}\right) i_{t}^{\prime} h^{t} \subseteq\left(\bigcap_{k=1}^{\infty} J^{k}\right) J h^{t}=\left(\bigcap_{k=1}^{\infty} J^{k}\right) h^{t} \subseteq I
$$

by (1) again for $t=2, \ldots, n-1$. It follows from (3) that

$$
\left(\bigcap_{k=1}^{\infty} J^{k}\right) h \subseteq I
$$

which contradicts (1). 


\section{AN EXample}

We conclude with an example which shows our generalisation is non-trivial.

EXAMPLE 3: Let $F$ be a field and $F[[x]]$ be the power series ring of $F$ over $x$. Then $F[1 / x]$, the polynomial ring over $1 / x$, is an Artinian $F[[x]]$-module, and the trivial extension, $R=F[[x]] \propto F[1 / x]$, is a commutative linearly compact ring which is not Noetherian (see [7, Example 10.9] for details). Let $J=x F[[x]] \times F[1 / x]$, which is the Jacobson radical of $R$. We see that ${ }_{R} J$ is finitely generated, $R\left(J / J^{k}\right)$ is Artinian for each $k \in \mathbb{N}$, and clearly $J$ is nilpotent over $J^{k}$.

\section{REFERENCES}

[1] F.W. Anderson and K.R. Fuller, Rings and categories of modules, 2nd edition (SpringerVerlag, Berlin, Heidelberg, New York, 1992).

[2] I.N. Herstein, 'A theorem on left Noetherian rings', J. Math. Anal. Appl. 15 (1966), 91-96.

[3] I.N. Herstein, 'A nil-nilpotent type of theorem', in Aspects of mathematics and its applications, (J.A. Barroso, Editor) (North-Holland, Amsterdam, 1986).

[4] C. Menini, 'Jacobson's conjecture, Morita duality and related questions', J. Algebra 103 (1986), 638-655.

[5] J.H. Meyer, 'A nil-implies-nilpotent result in Artinian rings', Bull. Austral. Math. Soc. 34 (1986), 267-269.

[6] J.T. Stafford, 'A nil implies nilpotent theorem for left ideals', J. Algebra 133 (1990), 545-549.

[7] Weimin Xue, Rings with Morita duality, Lecture Notes Math. 1523 (Springer-Verlag, Berlin, Heidelberg, New York, 1992).

\footnotetext{
Department of Mathematics

Fujian Normal University

Fuzhou

Fujian 350007

People's Republic of China
} 